\title{
Laser stimulated plasma-induced luminescence for on-air material analysis
}

S. Veltri, M. Barberio, C. Liberatore, M. Scisciò, A. Laramée, L. Palumbo, F. Legaré, and P. Antici

Citation: Appl. Phys. Lett. 110, 021114 (2017); doi: 10.1063/1.4973467

View online: http://dx.doi.org/10.1063/1.4973467

View Table of Contents: http://aip.scitation.org/toc/apl/110/2

Published by the American Institute of Physics

\section{Articles you may be interested in}

Optical properties of Cu2ZnSn(SxSe1-x)4 solar absorbers: Spectroscopic ellipsometry and ab initio calculations Appl. Phys. Lett. 110, 021905021905 (2017); 10.1063/1.4973353

Chiroptical properties photo-induced by femtosecond laser irradiation in silica glass

Appl. Phys. Lett. 110, 021112021112 (2017); 10.1063/1.4973198

Collimated gamma photon emission driven by PW laser pulse in a plasma density channel Appl. Phys. Lett. 110, 021102021102 (2017); 10.1063/1.4973972

Controlling surface plasmon polaritons at femtosecond timescales on an aluminum-coated grating Appl. Phys. Lett. 110, 021105021105 (2017); 10.1063/1.4973860

A designing principle for low dark-current strained layer superlattices Appl. Phys. Lett. 110, 021113021113 (2017); 10.1063/1.4974031

Stacking fault and defects in single domain multilayered hexagonal boron nitride Appl. Phys. Lett. 110, 023101023101 (2017); 10.1063/1.4972848

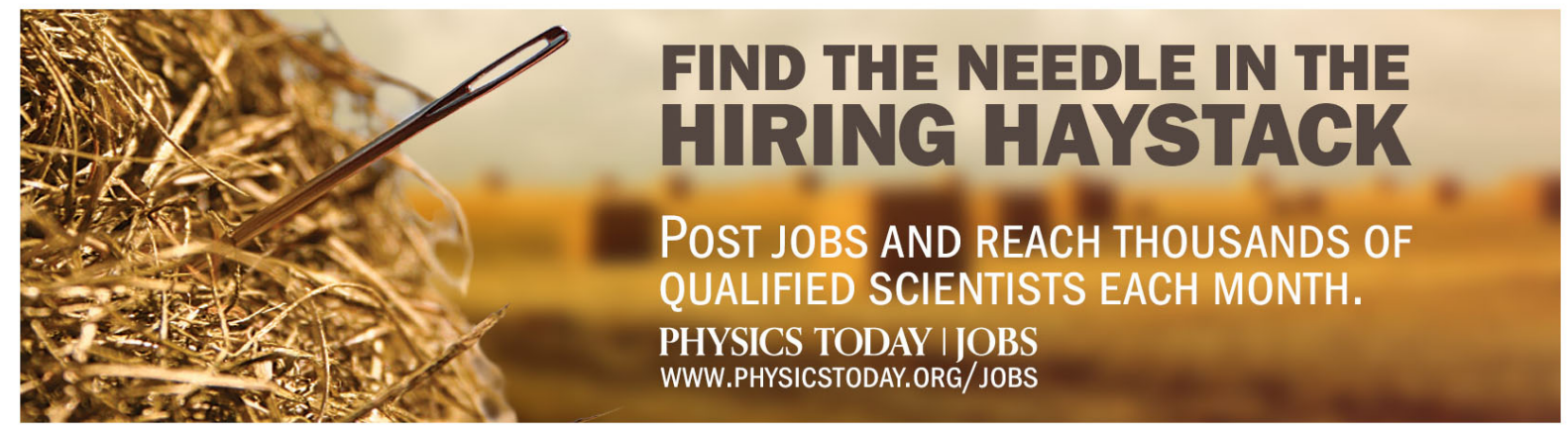




\title{
Laser stimulated plasma-induced luminescence for on-air material analysis
}

\author{
S. Veltri, ${ }^{1,2,3}$ M. Barberio, ${ }^{1,2,4}$ C. Liberatore, ${ }^{1}$ M. Scisciò, ${ }^{1,3}$ A. Laramée, ${ }^{1}$ L. Palumbo, ${ }^{3}$ \\ F. Legaré, ${ }^{1}$ and P. Antici ${ }^{1,3}$ \\ ${ }^{1}$ INRS-EMT, 1650 Boul. Lionel Boulet, Varennes, Quebec J3X 1S2, Canada \\ ${ }^{2}$ University of Calabria, via Bucci 4 B, 87036 Rende, CS, Italy \\ ${ }^{3}$ INFN University of Rome "La Sapienza" Piazzale A. Moro 5, 00185 Rome, Italy \\ ${ }^{4}$ Secondary Sources Divisions, ELI-ALPS, Tsiza Lajos krt, 85-87, 6720 Szeged, Hungary
}

(Received 26 October 2016; accepted 17 December 2016; published online 12 January 2017)

\begin{abstract}
In this work, we present a method for performing analysis of the chemical composition and optical properties of materials using In-Air Plasma-Induced Luminescence. This is achieved by interaction of a focused high-energy laser with air, an interaction that produces a sub-millimetric plasma. The energetic electrons generated and accelerated in the plasma at energies higher than $5 \mathrm{keV}$ reach the target surface of the sample to be analyzed, causing luminescence emission and plasmonic resonance. Each material is characterized by different chemical and optical properties that can be determined with the above-described technique. As such, our method allows obtaining an exact analysis of the sample, covering surfaces in the range of tens of $\mathrm{mm}^{2}$, in only a few minutes. We show that the acquired information with our method is identical to what obtained with more sophisticated methods, such as SEM-cathodoluminescence and photoluminescence. Published by AIP Publishing. [http://dx.doi.org/10.1063/1.4973467]
\end{abstract}

In the last decades, strong effort has been put into research of innovative techniques in the field of Physics and Chemistry applied to the introduction of innovative and low cost methods for the diagnostic of materials. Currently, many groups worldwide explore the possibility of developing improved diagnostics ${ }^{1,2}$ where the main challenge is to obtain the most information available without provoking damage to the materials. ${ }^{3}$

Chemical information on the materials is obtained using surface spectroscopies (such as Photoluminescence, Raman, X-ray photoelectron spectroscopy (XPS), X-Ray-Fluorescence (XRF), and Energy Dispersive X-ray Fluorescence (EDX) in SEM). The complete chemistry of the bulk material is retrieved using more sophisticated (and expensive) nuclear physics techniques such as Proton Induced X-ray and Gamma Emission (PIXE and PIGE). ${ }^{4,5}$

All these diagnostics present several limitations. Raman and photoluminescence spectroscopy techniques require sophisticated spectrometers and lasers. ${ }^{6}$ SEM and XPS must be taken under vacuum conditions. PIXE and PIGE require the employment of small conventional particle accelerators (typically ranging from a few $\mathrm{keV}$ to maximum a few $\mathrm{MeV}$ ) that are located in dedicated laboratories since their operation requires particular analysis conditions (e.g., ultra high vacuum conditions and strongly controlled temperature). ${ }^{7}$ Additionally, these tools are able to analyze directly only small surfaces (their beam spot sizes are generally in the order of $\mu \mathrm{m}^{2}$ ), which makes a complete analysis on a larger surface very time consuming (pencil-scanning analysis).

In this framework, luminescence methods (cathodoluminescence (CL), photoluminescence, and ionoluminescence $)^{8-12}$ assume particular relevance in different scientific fields (from mineralogy to semiconductor physics to nanotechnology) for their ability to identify the chemical composition of materials with a resolution of a few ppm and the individuation of plasmonic resonances (PR). Luminescent materials are mostly solid inorganic materials consisting of a host lattice with impurities. The impurity concentrations generally are low in view of the fact that at higher concentrations the efficiency of the luminescence process usually decreases (concentration quenching). The absorption of energy, which is used to excite the luminescence, takes place by either the host lattice or by impurities. In most cases, the emission takes place on the impurity ions, which, when they generate also the desired emission, are called activator ions. The luminescence emission can be induced by different methods (i.e., high energetic electron or ion irradiation, heating a sample to a temperature in the range between 300 and $600^{\circ} \mathrm{C}$, or by UV laser irradiation). While photoluminescence can be taken in air using a simple UV laser, most other methods such as cathodo- or iono-luminescence require a more complex setup, e.g., the need to be operated under SEM and vacuum conditions. Nevertheless, these latter methods are crucial for distinguishing structures, in particular when it comes to observe plasmon emission in metals or in nanostructured materials.

In this paper, we show that it is possible to perform cathode-luminescence measurements without using a SEM and stringent vacuum conditions. For this purpose, we introduce a laser-based in-air setup for obtaining a complete chemical analysis of the materials at its surface and within its bulk (within a depth of about $2 \mu \mathrm{m}$ from the surface) based on the study of luminescence emission induced by the plasma radiation (Plasma-Induced Luminescence, PIL). We use the beta radiation produced in the laser-plasma interaction for generating cathode-luminescence and plasmonic emission and from there obtain the chemical composition of the material is obtained. The electron energy produced during the laser-plasma interaction is in the order of a few tens of $\mathrm{keV}$. According to the NIST database, ${ }^{13}$ the mean free path in air for electrons with mean energy in the order of tens of $\mathrm{keV}$ is several centimeters; this guarantees that the 
produced electrons reach the sample surface with sufficient energy (up to the tens of keV level).

The effectiveness of our method is tested on three different materials with diverse density and chemical properties: metals (Gold), insulators (a scintillator with a layer of aluminum oxide with a thickness of about $500 \mathrm{~nm}$ deposited on an aluminum substrate), and minerals (Marble — a mineral composed by a matrix of $\mathrm{CaCO}_{3}$ with insertion of different impurities (such as $\mathrm{Mn}, \mathrm{Si}, \mathrm{Al}, \mathrm{Ca}, \mathrm{Fe}$, and $\mathrm{Ti}$ ) residuals of natural growth). We demonstrate that our method reveals the plasmonic emission from metals as well as the intrinsic luminescence of oxide or the emission induced by impurities in minerals. In addition, while conventional luminescence techniques are able to characterize only surface areas in the order of a few microns (invested area from laser, electron, or ion beams), Plasma-Induced Luminescence is able to analyze a surface area in the order of tens of $\mathrm{mm}^{2}$ in only a few minutes. At current, our setup requires a sophisticated laser system, similar to the case of Raman and photoluminescence. Nevertheless, several reasons are in favor of our approach:

(1) The aim of our paper is to investigate an in-air method for analysis of materials based on laser-plasma electron acceleration, which can be competitive and an alternative respect to usual methods applied in material science, in particular, for the analysis of macromaterials and macrostructures. (2) Laser-plasma electron acceleration is becoming a field in which more and more routinely particles can be accelerated with less expensive laser systems. Considering the current trend of high-power laser technology (and associated costs), it is not impossible that these laser-systems will soon be accessible at a more reasonable price and be competitive to other techniques.

The air plasma was generated by the interaction of a strongly focused-down high-energy laser, the Advanced Laser Light Source (ALLS) few-cycle infrared (IR) beamline, with air. The ALLS source provides a $3 \mathrm{~mJ}, 15$ fs laser pulse, centered at $1.8 \mu \mathrm{m}$ with a repetition rate of $100 \mathrm{~Hz}$. The energy stability of the pulse is approximately $2.5 \%$ rms. ${ }^{14}$ The experimental setup, shown in Figures 1(a) and 1(b), consists of a series of transport optics, an on-axis parabola, and a series of imaging lines for the plasma analysis and luminescence collection.

The luminescence emission was stimulated by the portion of plasma radiation collected through a hole with a diameter of $6 \mathrm{~mm}$ placed at a distance of $6 \mathrm{~mm}$ from the center of the plasma. The investigated area on the target surface can be modulated by changing the dimension of the hole, allowing analyzing areas from a few $\mu \mathrm{m}^{2}$ to $\mathrm{cm}^{2}$. Luminescence emission at an angle of $45^{\circ}$ was collected by a lens positioned at $4.5 \mathrm{~cm}$ from the irradiated target (focal distance) and transferred through an optical fiber to a spectrometer working in the UV-Visible (VIS) range (VIS-140 Horiba). Each luminescence spectrum was collected with an acquisition time of $120 \mathrm{~s}$ in order to allow for a sufficient signal/noise ratio.

All the experiments were conducted in darkness for eliminating possible sources of background light that would add noise to the measurement. We irradiate three samples (gold, aluminum oxide, and marble) that have a well-known luminescence emission stimulated for both, photons and electrons. This allows a direct comparison of our results with those present in literature and from there to attribute the emitted luminescence to different components within the plasma radiation (all the data will be compared with established luminescence data bases (see Ref. 14 and reference therein).

The plasma produced in the interaction between the laser beam and air is visible in optical images shown in Figure 1(a). The plasma displays a spheroidal shape with axes of 2 and $3 \mathrm{~mm}$ and a photon emission in the visibleNear Infra-Red (NIR) region with 5 lines at 1.5, 1.97, 2.12, 2.23 , and $2.44 \mathrm{eV}$. The absence of an UV line in the plasma excludes the possibility of photoluminescence emission coming from the target sample. Furthermore, a Faraday cup reads
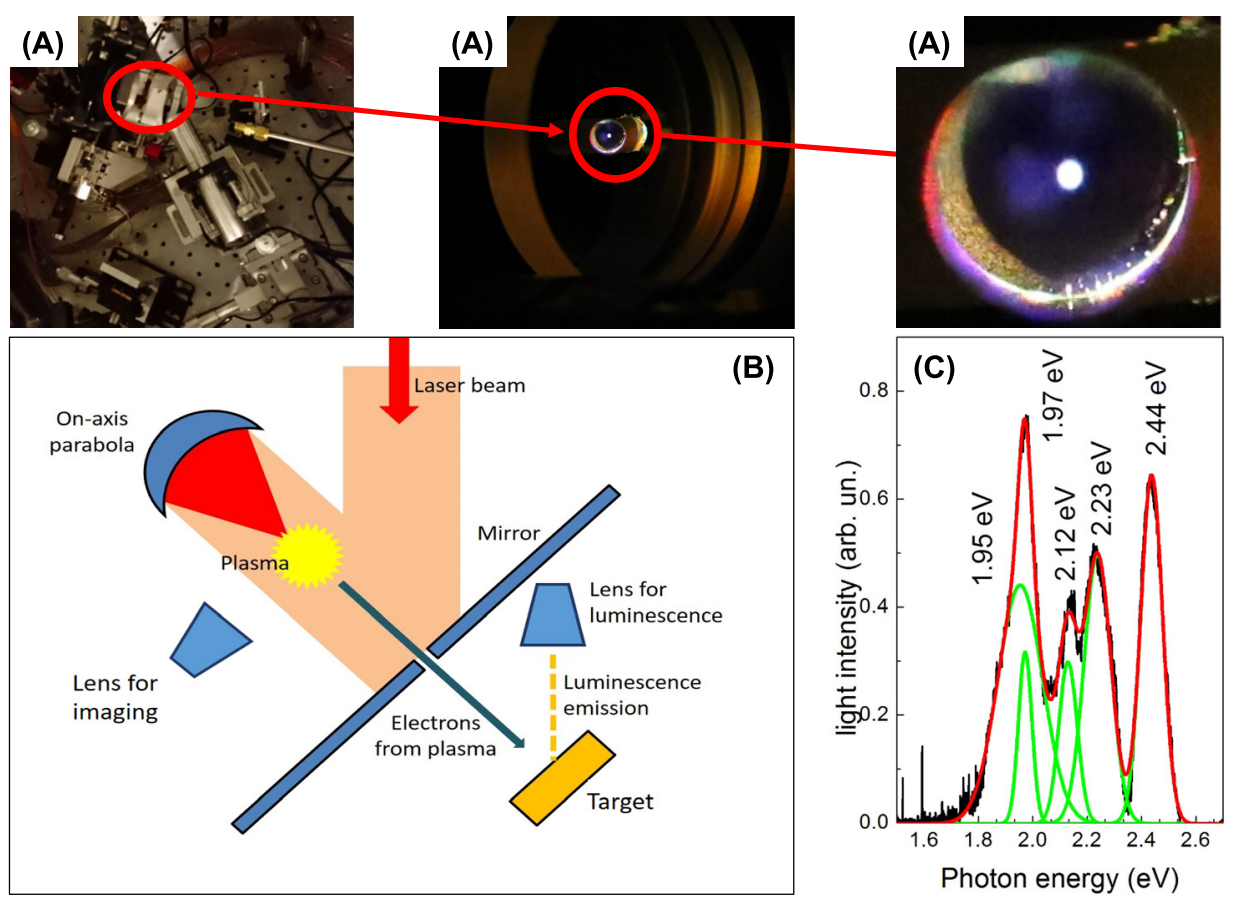

FIG. 1. (a) Photographs of the experimental setup for air plasma generation with details about the mask protecting the sample, (b) sketch of experimental setup for the plasma-induced luminescence generation and analysis, and (c) spectra of the photon emission obtained from the plasma. The emission is concentrated in the VIS-NIR region, excluding the possibility of photoluminescence generation from the target surface. The red curves indicate the multi-peak Gaussian fit while the green curves indicate the single Gaussian curves included in the fit. 
a negative current of about $40 \mathrm{pA}$ in the entire region around the plasma, at a distance of up to $20 \mathrm{~cm}$ from the plasma center, indicating the presence of electrons produced in the plasma and isotropically accelerated.

The first evidence of air-plasma-induced luminescence is visible in Figure 2(a) where the image of the plasma, through a slit, was taken on the surface of the aluminum oxide sample. The green-red colored emission shows a spectrum composed by 10 bands (summarized in Table I) typical of aluminum cathodoluminescence (CL) and plasmonic resonance (PR). In particular, the $237,333,403,507,750$, and $770 \mathrm{~nm}$ bands are attributed to the CL emission generated from intrinsic defects (anion vacancies) present on the surface of the aluminum oxide sample (see Ref. 15 and references therein). To be noted that the aluminum oxide sample we used was composed by a thin layer of aluminum oxide (thickness in the order of $500 \mathrm{~nm}$ ) deposed onto an aluminum bulk. Electrons with a mean energy of 5-20 keV penetrate into the bulk up to a depth of a few microns, therefore investigating both, the aluminum oxide layer and the aluminum structure. This explains some spectral features of metallic aluminum in the aluminum oxide spectrum. The characteristic lines for all these elements can be found in literature only when observed under CL-SEM conditions; considering that a SEM works with electron energy above $20 \mathrm{keV}$, this indicates that our findings are induced by electrons with at least this energy. ${ }^{14}$ The 540, 576, and 670 bands are attributed to plasmonic resonance on the aluminum, generated by electrons with a minimum energy of $5 \mathrm{keV} .{ }^{16}$ EDX spectra (Figure 2(c)) of the scintillator indicate merely the presence of $\mathrm{Al}, \mathrm{O}$, and $\mathrm{C}$ on the surface, confirming the equivalence of the luminescence bands. Similar results are obtained for all the other materials (see Figure 3). Luminescence of the marble (Figure 3(a)) reveals six bands in the region 300-600 $\mathrm{nm}$.
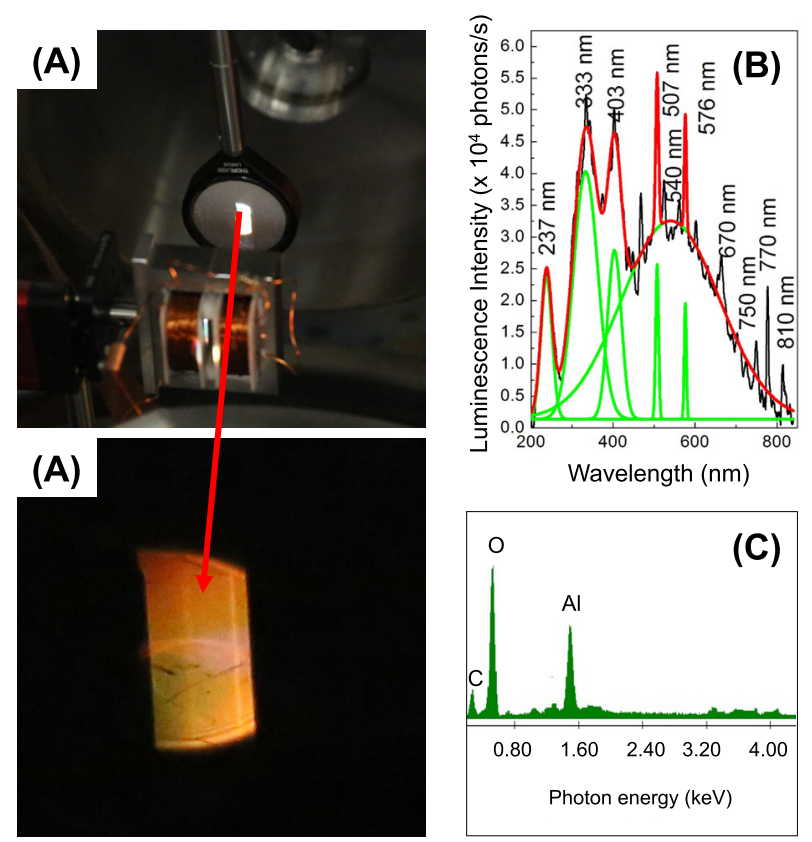

FIG. 2. Luminescence emission from the aluminum oxide sample; (a) photographs showing the emission, (b) spectrum, and (c) EDX analysis of chemical composition. The red curves indicate the multi-peak Gaussian peak while the green curves indicate the single Gaussian curves included in the fit.
TABLE I. Summary of plasma-induced luminescence bands.

\begin{tabular}{lcc}
\hline \hline Gold $(\mathrm{nm})$ & Aluminum $(\mathrm{nm})$ & Marble $(\mathrm{nm})$ \\
\hline 530 & 237 & 305 \\
599 & 333 & 337 \\
& 403 & 398 \\
& 507 & 519 \\
& 540 & 506 \\
& 576 & 577 \\
& 670 & \\
& 750 & \\
& 770 & \\
\hline \hline
\end{tabular}

All the bands can be attributed to CL-SEM ( $>20 \mathrm{keV})$ emission from intrinsic defects (lattice vacancies) or to $\mathrm{Mn}^{2+}$ external impurities (the presence of Mn impurities was confirmed by EDX spectra in Figure 3(b)). ${ }^{14}$ We can exclude the presence of photoluminescence emission due to the absence of bands in the range from 600 to $700 \mathrm{~nm}^{14}$ On the other hand, the plasma spectrum (Figure 1(c)) does not show photons in the UV region $(>3 \mathrm{eV})$. Luminescence emitted from the pure gold target (see Figure 3(c) for a luminescence spectrum and Figure 3(d) for the EDX analysis) indicates the presence of two bands at 530 and $599 \mathrm{~nm}$, both attributed to gold plasmon resonance induced by irradiation of electrons ranging from $5 \mathrm{keV}$ to $30 \mathrm{keV} .^{17,18}$ The efficiency of electron-tophoton conversion for the plasmon emission from gold (for the plasmons at $530 \mathrm{~nm}$ and $599 \mathrm{~nm}$, and considering electrons with an energy between 5 and $30 \mathrm{keV}$ ) is about $10^{-4}$ photons per incident electron. ${ }^{16}$ The maximum photon number calculated from the luminescence spectra is about $2.5 \times 10^{4}$ photons/s (see Figure $3(\mathrm{c})$ ); this yields to an electron incidence of about $2.5 \times 10^{8}$ electrons $/ \mathrm{s}$, which is close to the current measured by the Faraday cup (about $40 \mathrm{pA}$ ).
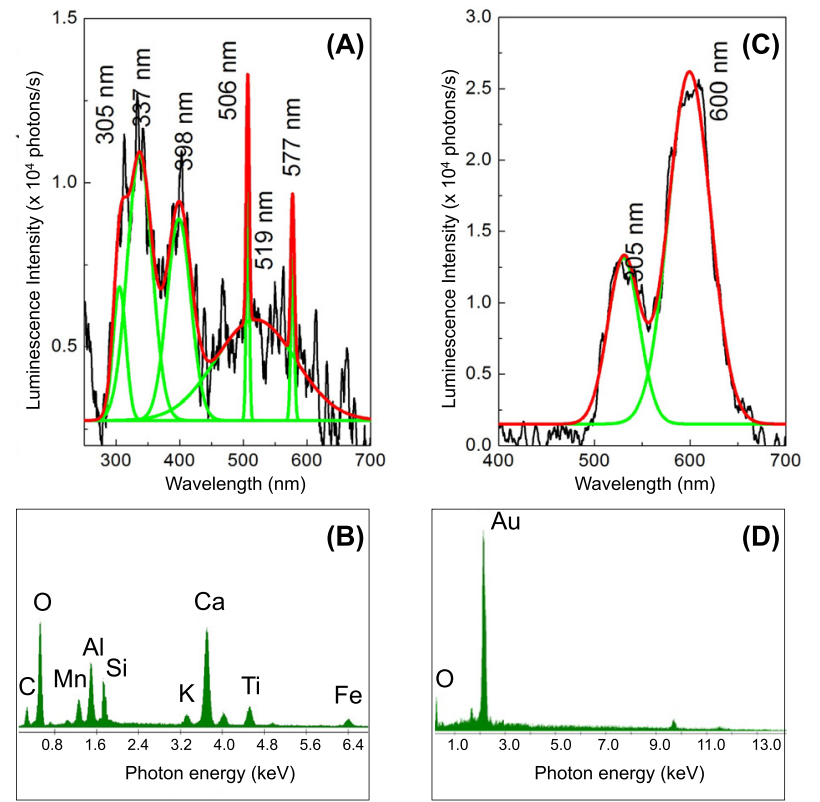

FIG. 3. Luminescence emission and chemical composition for the Marble (a) and (b) and Gold target (c) and (d). The red curves indicate the multipeak Gaussian peak while the green curves indicate the single Gaussian curves included in the fit. 
The exact correspondence between the measured electron current on the sample surface and the photon rate/production clearly indicates that the plasma-induced luminescence can exclusively be attributed to the electrons produced and accelerated within the plasma.

In this work, we demonstrated that it is possible to generate luminescence from materials exposed to a laser generated air-plasma, obtaining a precise analysis of the material composition. The results related to the chemical composition of the materials obtained with our technique are identical to those of more complicated and complex methods such as SEM cathodoluminescence, photoluminescence, and analysis of plasmonic resonance. We test three different materials, aluminum oxide, gold, and marble, confirming that for all materials plasma-induced luminescence is able to induce luminescence emission from structural defects and plasmon resonance. Plasma-induced luminescence is attributed to the interactions between the electrons produced and accelerated around the plasma (in the laser-air interaction) and the sample material. Finally, if one estimates the electron energy to be between 5 and $30 \mathrm{keV}$, one is able to investigate within 2 min a large surface in the tens of $\mathrm{mm}^{2}$ range with a penetration depth varying from $2 \mu \mathrm{m}$ for the gold and marble samples up to $6 \mu \mathrm{m}$ for the aluminum oxide sample.

We thank J. C. Kieffer, S. Payeur, and S. Fourmaux for their support and P. Sapia for borrowing the spectrometer and useful discussions. This work was supported by FRQNT (nouveaux chercheurs, Grant No. 174726, Equipe 2016-PR189974) and NSERC Discovery Grant (Grant No. 435416).

\footnotetext{
${ }^{1}$ P. Baglioni, E. Carretti, and D. Chelazzi, "Nanomaterials in art conservation," Nat. Nanotechnol. 10, 287-290 (2015).

${ }^{2}$ M. Bawaya, "Salvaging science," Science 347(6218), 117 (2015).

${ }^{3}$ G. Chiari, "Saving art in situ," Nature 453, 159 (2008).
}

${ }^{4}$ A. D. Carbó, "Electrochemistry for conservation science," J. Solid State Electrochem. 14, 349-351 (2010).

${ }^{5}$ N. Grassi, A. Migliori, P. A. Mandò, and H. Calvo del Castillo, "Differential PIXE measurements for the stratographic analysis," X-ray Spectrom. 34, 306 (2005).

${ }^{6}$ M. Giarola, G. Mariotto, M. Barberio, and D. Ajò, "Raman spectroscopy in gemmology as seen from a 'jeweller's' point of view," J. Raman Spectrosc. 43, 1828 (2011); M. Schreiner, M. Melcher, and K. Uhlir, "Scanning electron microscopy and energy dispersive analysis: Applications in the field of cultural heritage," Anal. Bioanal. Chem. 387, 737 (2007).

${ }^{7}$ Special Issue: "Science and Technology of Cultural Heritage Materials: Art Conservation and Restoration"; edited by M. Stuke, G. Chiari, C. Fotakis, M. Menu, and G. Padeletti, Applied Physics A (2008), vol 92, issue 1; J. Salomon, J.-C. Dran, T. Guillon, B. Moignard, L. Pichon, P. Walter, and F. Mathis, "Present and future role of ion beam analysis in the study of cultural heritage materials: The example of the AGLAE facility," Nuclear Instruments and Methods in Physics Research B 266, 2273-2278 (2008).

${ }^{8}$ M. Barberio, P. Barone, A. Bonanno, M. Camarca, and F. Xu, "Thermoand iono-luminescence on MWCNT bundles," Radiat. Phys. Chem. 76(3), 492 (2007).

${ }^{9} \mathrm{M}$. Barberio, P. Barone, and A. Oliva, "Optical properties of ZnS-carbon nanotubes composites," Radiation Phys. Chem. 81(6), 642 (2012).

${ }^{10} \mathrm{C}$. Ronda, Luminescence: From Theory to Applications (Wiley-VCH Verlag GmbH \& Co. KGaA, 2008).

${ }^{11}$ C. Furetta, Handbook of Cathodoluminescence (World Scientific, 2010), ISBN: 978-981-238-240-5.

${ }^{12}$ H. S. Nalwa and L. S. Rohwer, Handbook of Luminescence, Display Materials and Devices (American Scientific Publishers - Chemistry) ISBN: 1-58883-010-1.

${ }^{13}$ See https://www.nist.gov/srd/nist-standard-reference-database-71 for NIST reference database.

${ }^{14}$ S. Payeur, S. Fourmaux, B. E. Schmidt, J. P. MacLean, C. Tchervenkov, F. Légaré, M. Piché, and J. C. Kieffer, "Generation of a beam of fast electrons by tightly focusing a radially polarized ultrashort laser pulse," Appl. Phys. Lett. 101, 041105 (2012).

${ }^{15}$ See http://www.csiro.au/luminescence/ for Luminescence database.

${ }^{16}$ M. W. Knight, N. S. King, L. Liu, H. O. Everitt, P. Nordlander, and N. J. Halas, "Aluminum for plasmonics," ACS Nano 8(1), 834-840 (2014).

${ }^{17}$ T. Coenen, E. Jan, R. Vesseur, and A. Polman, "Angle-resolved cathodoluminescence spectroscopy,” Appl. Phys. Lett. 99, 143103 (2011).

${ }^{18}$ F. J. García de Abajo, "Optical excitations in electron microscopy," Rev. Mod. Phys. 82, 209 (2010). 